\title{
Online Calibration and Performance of the ATLAS Pixel Detector
}

\author{
M. Keil ${ }^{\mathrm{a}, \mathrm{b}, 1}$ \\ ${ }^{a}$ CERN, 1211 Geneva 23, Switzerland \\ ${ }^{b}$ II. Physikalisches Institut, Universität Göttingen, \\ Friedrich-Hund-Platz 1, 37077 Göttingen, Germany
}

\begin{abstract}
The ATLAS Pixel Detector is the innermost detector of the ATLAS experiment at the Large Hadron Collider at CERN. It consists of 1744 silicon sensors equipped with approximately 80 million electronic channels, providing typically three measurement points with high resolution for particles emerging from the beaminteraction region, thus allowing measuring particle tracks and secondary vertices with very high precision. The readout system of the Pixel Detector is based on a bi-directional optical data transmission system between the detector and the data acquisition system with an individual link for each of the 1744 modules. Signal conversion components are located on both ends, approximately $80 \mathrm{~m}$ apart.

This paper describes the tuning and calibration of the optical links and the detector modules, including measurements of threshold, noise, charge measurement, timing performance and the sensor leakage current.
\end{abstract}

\section{Introduction}

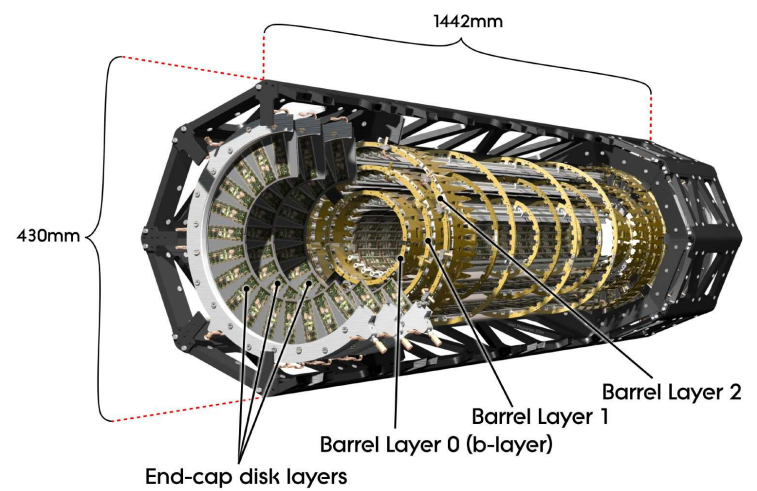

Figure 1: Schematical drawing of the ATLAS Pixel Detector. The detector comprises three barrel layers and two endcaps with three discs each. The individual detector modules are mounted on carbon fibre support structures with incorporated cooling circuits.

The ATLAS Pixel Detector [1, 2], shown in Fig. 1, 27 is the innermost tracking detector of the ATLAS

\footnotetext{
${ }^{1}$ On behalf of the ATLAS collaboration.
}

experiment [2]. It is made of three concentric barrel layers with mean radii of $50.5 \mathrm{~mm}, 88.5 \mathrm{~mm}$ and $122.5 \mathrm{~mm}$ centred around the beam axis and two endcaps with three discs each, forming a threehit system up to pseudo-rapidities of \pm 2.5 . The full detector contains 1744 pixel modules, which are mounted on carbon fibre local supports. An evaporative $\mathrm{C}_{3} \mathrm{~F}_{8}$ cooling system is incorporated into the local supports to absorb the heat produced by the modules and to allow for an operation at temperatures below $0^{\circ} \mathrm{C}$, to limit the effects of radiation damage.

The individual pixel modules (Fig. 2) are made of a $250 \mu \mathrm{m}$ thick n-on-n silicon sensor, 16 front-end chips and a module controller chip (MCC) [3]. The sensor is divided into 47,232 pixels with a typical pixel size of $50 \mu \mathrm{m} \times 400 \mu \mathrm{m}$; approximately $10 \%$ of pixels have a size of $50 \mu \mathrm{m} \times 600 \mu \mathrm{m}$ (long pixels) to bridge the gaps between the readout chips in the long pixel direction. Another 2.5\% of the electronics channels have two sensor pixel connected to bridge the gaps in the other direction (ganged pixels). The sensor is read out by 16 front-end chips with 2880 electronics channels each. Each pixel cell contains a charge sensitive preamplifier, a discriminator and the necessary digital readout logic to transfer hits to the peripheral circuitry of the chip, the end-of-column (EOC) logic. In the EOC logic 
hits are stored up to the programmable trigger latency and sent to the module controller chip in case a trigger arrives at the correct latency, erased otherwise. Together with the mere hit location and time, the time-over-threshold (ToT) information is read out for each hit. This is the time interval during which the preamplifier output is above the threshold, in units of the bunch crossing clock (25 ns). Due to the pulse shape of the preamplifier the ToT is a nearly linear function of the deposited charge. Evaluating this ToT information can therefore be used to infer the charge deposited by a passing particle. The routing of signals and power lines of the module is done on the flex hybrid. This is a flexible polyimide PCB, which is glued onto the backplane of the sensor. The connection to the front-end chip is made with wire bonds. The flex hybrid also carries the MCC, which controls the front-end chips and performs an event building with the hit data received from the front-end chips. The off-module connection is provided by a micro cable (type-0 cable), which is either soldered directly onto the flex hybrid (in case of the disc modules) or connected to a polyimide pigtail (in case of the barrel module). Figure 2 shows the elements of a pixel barrel module.

The transmission of fast signals (data, clock and commands) between the detector and the readout crates in the counting rooms is achieved via optical links. One TTC link per module (Timing, Trigger and Commands) carries the clock and commands to the detector, a data link brings the hit data from the detector to the readout crates. Most modules have one associated data link, only the modules in the innermost barrel layer have two data links per module to accommodate the higher data rate. The electro-optical conversion on the detector side is done on optoboards, which are located at a distance of approximately $1 \mathrm{~m}$ from the interaction point. Figure 3 shows the overall readout scheme of the ATLAS Pixel Detector, from the sensor to ${ }_{81}$ the off-detector electronics in the counting room.

\section{Calibration Measurements}

\subsection{Tuning of the Optical Links}

In order to establish the communication between the detector modules and the off-detector electronics in the counting room, the optical links need to be tuned [4]. The most critical parameters are the threshold and the sampling phase of the

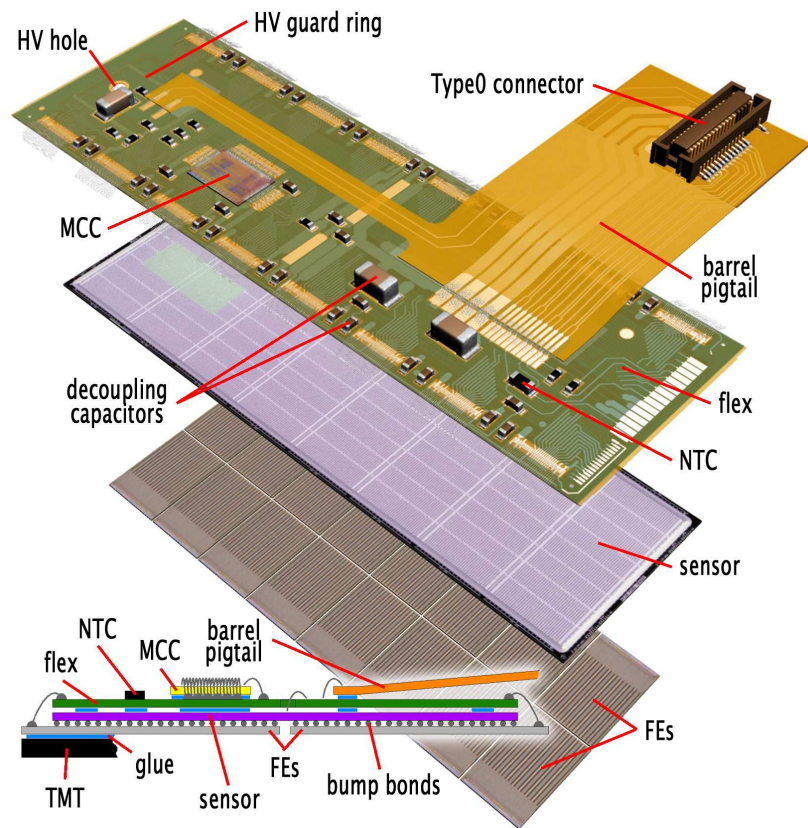

Figure 2: Assembly view and cross section of an ATLAS Pixel Detector module. Sixteen front-end chips are bump bonded to the silicon pixel sensor. Interconnections are done on a flexible polyimide PCB, which is connected by wire bonds to the electronics chips.

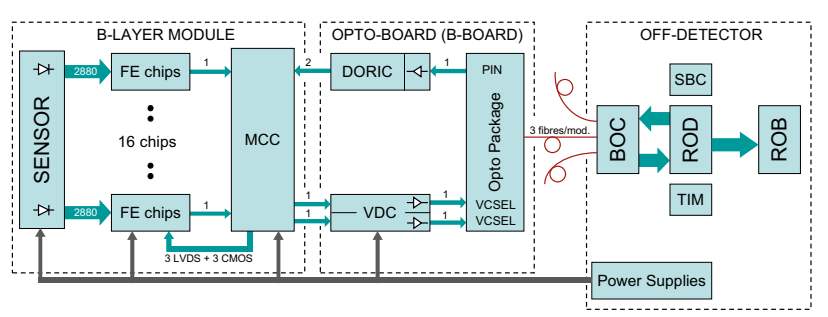

Figure 3: Block diagram of the ATLAS Pixel Detector readout system architecture.

uplink, which sends the hit data from the module to the readout crates. The correct settings of these parameters for each module are determined in the so-called BOC scan. This scan performs a bit error rate measurement, scanning the full twodimensional parameter space of delay and threshold. A typical scan result is shown in Fig. 4. White areas indicate no errors, all remaining regions have non-zero error rates. One sees an error floor for low thresholds, indicating noise which is interpreted as logical one, due to a too low threshold setting. The vertical error band corresponds to a sampling at a 


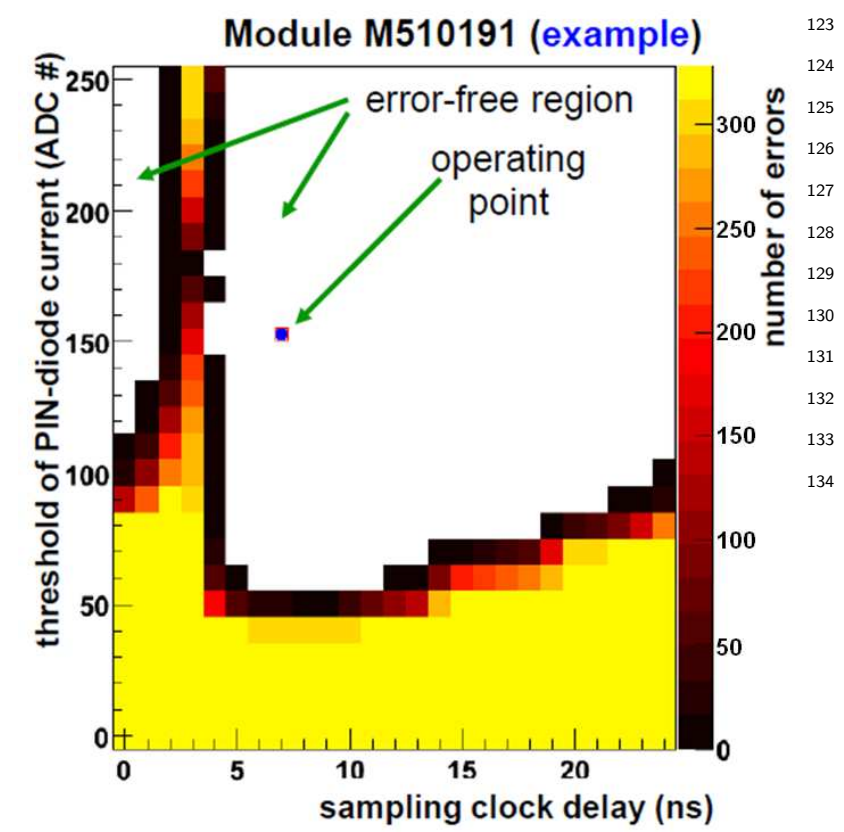

Figure 4: Scan of the optical link. The arrows indicate the error free region (white) and a possible set point for threshold and delay.

moment when the data transmitted is not stable ${ }_{117}$ but changes from one clock cycle to the next. An ${ }_{118}$ optimal operating point is chosen within the error 119 free regions, taking into account also possible fluc- ${ }_{120}$ tuations of the different error regions.

In case of need the laser power of the on-detector lasers can be adjusted. However the modularity for this adjustment is 6 or 7 modules, depending on the exact location of the modules. For the inner layers and the discs a similar tuning is performed at a readout speed of $80 \mathrm{MBit} / \mathrm{s}$. Currently all tunable ${ }^{135}$ modules (i.e. modules without more serious problems of the optical links) have been tuned without problems.

\subsection{Threshold and Noise}

The electronics parameters of the front-end pixel ${ }_{142}$ cells are assessed by means of a programmable ${ }_{143}$ charge injection circuit in the front-end chip. The ${ }_{144}$ timing and the amount of charge can be freely cho- ${ }_{145}$ sen, allowing to perform all necessary tunings and ${ }_{146}$ a wide range of measurements. To measure the ${ }_{147}$ threshold and noise values, injections are performed ${ }_{148}$ with charges around the expected threshold and for 149 each charge the number of hits for a fixed number 150 of injections are counted. The values of threshold and noise are then extracted from an error function fit to the obtained response curve [5].

In order to set the thresholds as homogeneously as possible, a similar scan is performed; however for this so-called threshold tuning the injected charge is fixed to the target threshold value and in each pixel the setting of the tune-DAC, which determines the discriminator threshold, is varied. The setting for which the response fraction is as close as possible to $50 \%$, is the ideal setting for the given target threshold [5].

Figure 5 shows the measured threshold values of all scanned pixels in the detector, after a tuning with a target threshold of $3500 \mathrm{e}$. We see that most pixels are well-centred around the target threshold value, the typical RMS of the threshold distribution after tuning is $\sim 40 \mathrm{e}$.

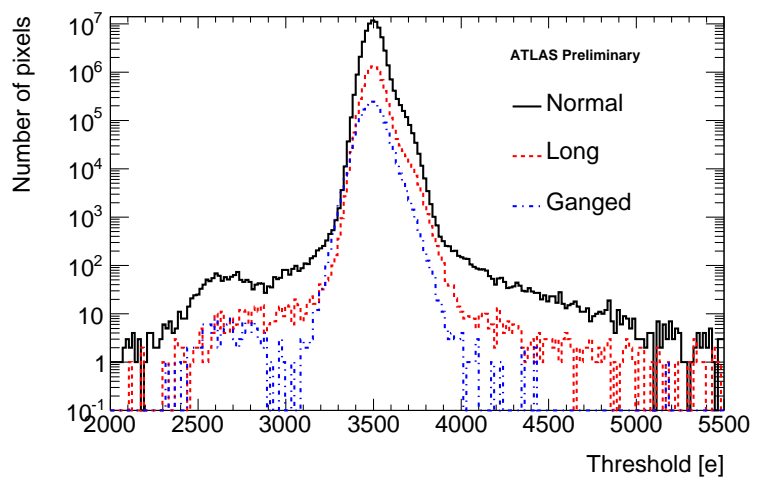

Figure 5: Measured threshold values for all scanned pixels in the detector. Prior to the scan the threshold settings have been tuned to $3500 \mathrm{e}$.

The noise values obtained from the same threshold scan are shown in Fig. 6. The behaviour is clearly different between the normal pixels and the pixels connected to the long or ganged sensor pixels at the edges of the front-end chips. This expected effect is due to the higher load capacitance those sensor pixels pose to the electronics. Typical noise values vary between $\sim 180 \mathrm{e}$, for the normal pixels and $\sim 300$ e for the class of the ganged pixels. In terms of the ratio between threshold and noise this leads to values of $\sim 20$ for the normal pixels and $\sim 12$ for the ganged pixels.

The noise occupancy observed in data taking is shown in Fig. 7. For data taking pixels exceeding a noise occupancy of $10^{-5}$ hits/bunch crossing (BC) are masked already in the module configu- 


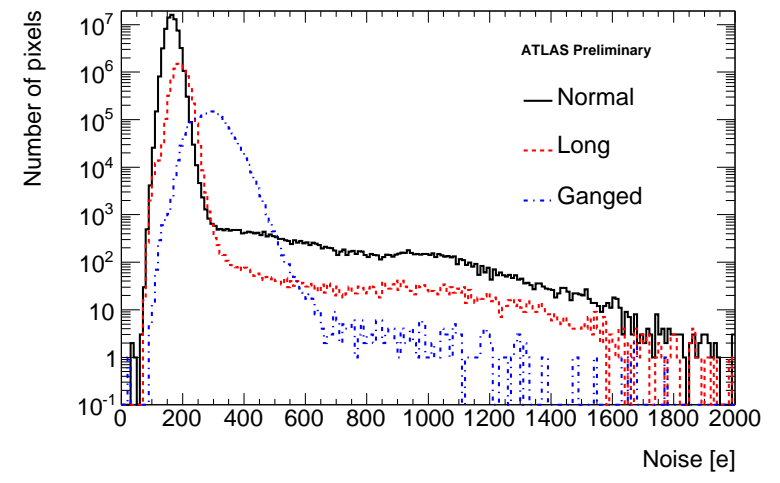

Figure 6: Noise values obtained from the threshold scan shown in Fig. 5

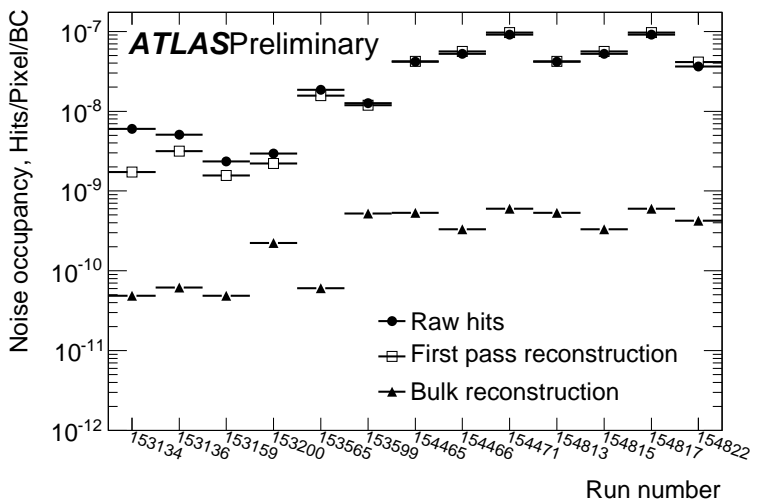

Figure 7: Noise occupancy vs. run number for a period of data taking. A small fraction of noisy pixels is masked online in the module configuration, a second group of noisy pixels 187 offline, before the bulk reconstruction.

ration. These pixels are determined in dedicated noise data taking runs and at a threshold setting of 3500 e typically amount to $0.1 \%$ of all pixels. On a run-by-run basis remaining noisy pixels (typically $0.01 \%-0.02 \%$ of all pixels) are determined in the first-pass reconstruction and then masked offline for the bulk reconstruction. After masking the noise occupancy is in the order of $10^{-10} \mathrm{hits} / \mathrm{pixel} / \mathrm{BC}$, corresponding to well below 1 hit per event in the

\subsection{Charge Measurement}

As described above, the ToT information stored 193 together with each hit can be used as a measure 194 for the deposited charge. In order to use the ToT ${ }_{195}$ full detector.
5 for the charge measurement it has to be calibrated. This is done in a similar manner to the threshold scan, but with an enlarged charge range, using charges above threshold up to twice the charge of a minimum ionising particle (mip). The curve of average ToT vs. injected charge is fit with a simple, invertible function, which is then used in the offline reconstruction to calculate the charge from the measured ToT values. To save storage space, only one calibration function is saved per frontend chip and pixel-type. It is therefore desirable that the ToT response is as homogeneous as possible between the pixels of a front-end chip. This is achieved by tuning the feedback current with a global 8-bit DAC on each front-end chip and an additional 3-bit DAC in each pixel cell.

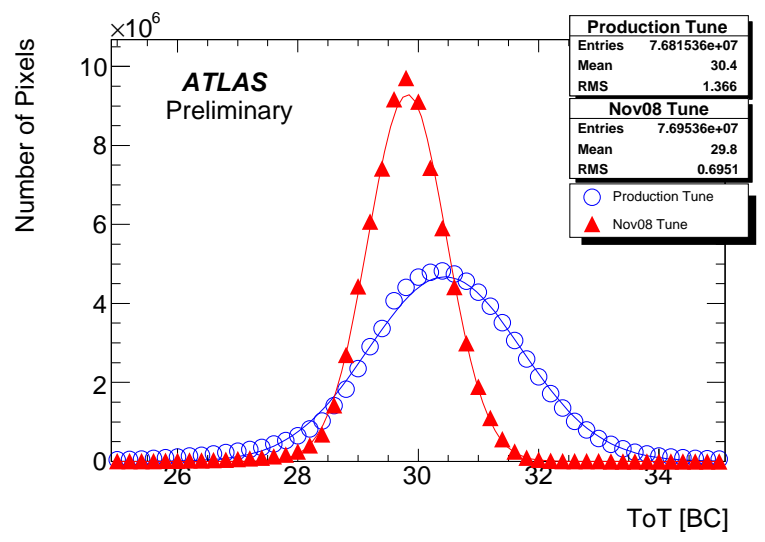

Figure 8: Average ToT for a 20 ke charge injection in all pixels of the detector. The open circles show the distribution using an "old" configuration from production scans, the closed triangles the distribution after re-tuning in the experiment.

Figure 8 shows the measured ToT values for all pixels in the detector for an injected charge of one mip. The figure compares the values measured after tuning in the experiment and those obtained with a configuration determined from a production tuning (The RMS for completely untuned modules is significantly larger, typically between 2 and 3 bunch crossings). The plot shows that after tuning the ToT is close to the target value of 30 bunch crossings, with an RMS of less than 1 bunch crossing. Figure 9 shows a charge spectrum determined from the ToT information. The comparison between simulation and data shows that the ToT method proA a reasonable measure of the deposited charge. A slight systematic disagreement on the order of 
up to two percent is not unexpected, as this is the 226 precision of the calibration of the injected charge, 227 which is used for the ToT calibration.

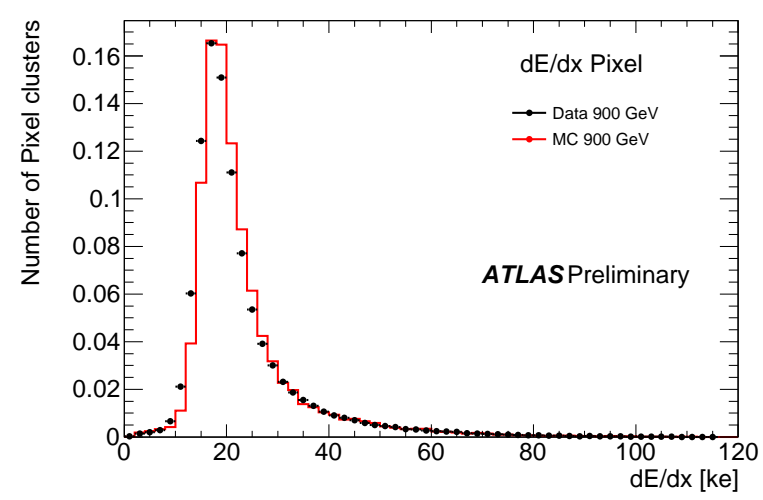

Figure 9: Cluster charge determined from the ToT information during data taking with $900 \mathrm{GeV}$ center-of-mass p-p collisions. The charges have been corrected for the different path lengths in the sensor according to the track incidence angle.

\subsection{Timing}

For an efficient reconstruction, the hits of particle tracks not only need to be detected, but also assigned to the correct interaction. This requires a time resolution of better than $25 \mathrm{~ns}$. On the front-end level the timing accuracy is limited by the timewalk, i.e. the effect that the preamplifier pulses for small charges cross the discriminator threshold later than those for large charges. This leads to an uncertainty in the detection time for different signals belonging to the same collision. The critical quantity to quantify this effect is the socalled in-time threshold, i.e. the smallest charge that is detected within the same bunch crossing as 244 a large reference charge. A measurement of these 245 in-time threshold values for all pixels in the detector 246 is shown in Fig. 10. The average in-time threshold 247 is 4800 e, approximately 1300 e above the discrimi- 248 nator threshold of 3500 e. Charges between 3500 e 249 and 4800 e are detected but not assigned to the ${ }_{250}$ correct bunch crossing. This behaviour can be im- 251 proved by a hit-doubling mechanism implemented 252 in the FE-I3 chip. This mechanism uses the ToT ${ }_{253}$ information of each hit to determine whether it has 254 suffered a timewalk of more than 1 BC. In case the 255 ToT is below a programmable threshold the hit is 256 written twice into the buffers, once with unchanged ${ }_{257}$ timing information and once one BC earlier. This mechanism allows to reduce the in-time threshold to a value 200 - 300 e above the discriminator threshold, at the cost of a slight $(\sim 10 \%)$ increase in the occupancy.

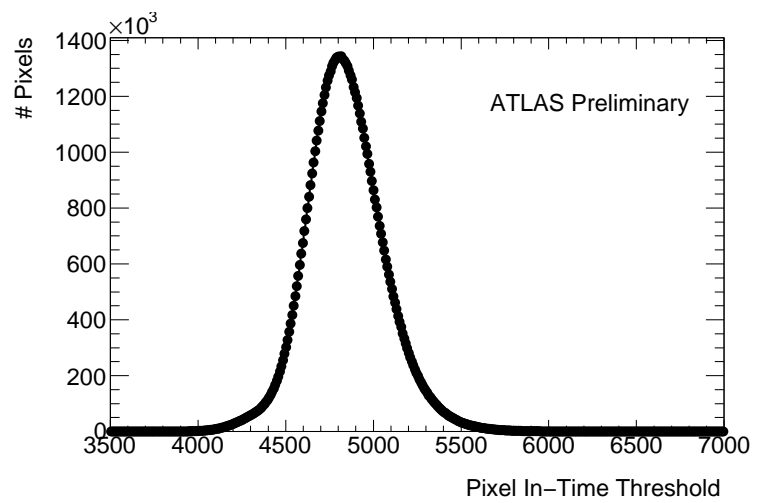

Figure 10: In-time threshold for all pixels in the detector, at a discriminator threshold setting of 3500 e.

On the system level both the homogeneity between the modules and the stability of the timing have been examined. To improve the homogeneity a sub-ns delay can be added to the clock and data lines of each individual module. This has been done, taking into account the different cable delays and the different time-of-flight for the different parts of the detector. After a final adjustment using collision data, a measurement of the detection time for large charges yields an RMS of 0.007 BC or 175 ps. This remaining module-to-module variation is in the order of the step width of the delay used for adjustment and therefore expected.

The stability of the module timing can be assessed from the stability of the vertical error band in the BOC scan (Fig. 4). Evaluation of several BOC scans over a period of 3 weeks has shown that for nearly all modules the maximal variation is below 2 ns. Both the module-to-module variations and the variations over time are tolerable as a $5 \mathrm{~ns}$ safety margin has been considered in the timing settings: the delay settings are such that the detection time of a large charge, i.e. a charge not suffering timewalk, is 5 ns after the beginning of the clock cycle. This leaves 20 ns margin for timewalk and $5 \mathrm{~ns}$ for random variations of the detection time, well above the experimentally observed variations. 


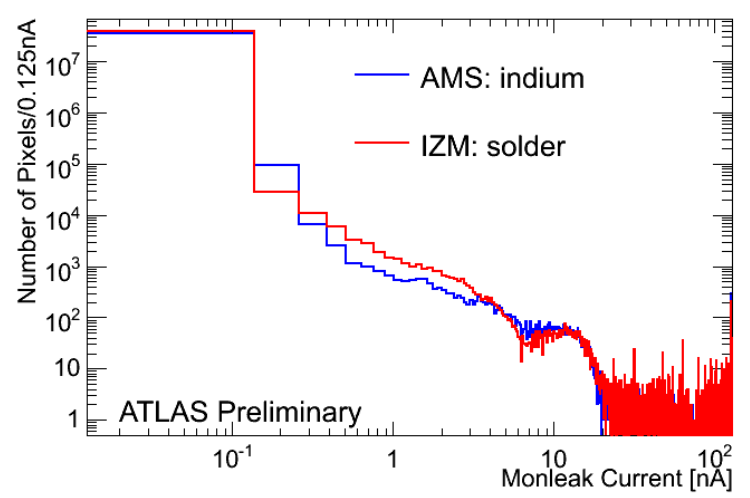

Figure 11: Measurement of the single pixel leakage current

for all pixels in the detector.

\subsection{Leakage Current Measurement}

The analogue pixel cells in the front-end chip contain the possibility to mirror the current in the ${ }^{29}$ feedback branch to the input of an on-chip ADC. This allows to measure the leakage current of each ${ }_{292}$ single sensor pixel. A measurement for all pixels in the detector is shown in Fig. 11. As the ADC ${ }^{293}$ range has been optimised for currents after irradia- ${ }^{29}$ tion, with a least significant bit of $\sim 0.125 \mathrm{nA}$, the leakage current is below the measurement sensitiv- 297 ity for most of the pixels, as expected. Together ${ }^{298}$ with other measurements, like measurements of the bump connectivity, this measurement will be reg- 30 ularly performed on the entire pixel detector, to 302 monitor the ageing of the detector and to compare the actual behaviour with the expectations.

\section{Summary}

Since its commissioning in 2008, the ATLAS Pixel Detector has been tuned and calibrated regularly. The performance in these calibrations is as expected and promises a successful operation of the detector in collision data taking.

The low threshold dispersion of $\sim 40$ e and the electronic noise of below 200 e lead to a noise occupancy in the order of $10^{-10} \mathrm{hits} / \mathrm{pixel} / \mathrm{BC}$. At a threshold setting of 3500 e the in-time threshold value is currently at approximately $4800 \mathrm{e}$. However, work to decrease this to below 4000 e is ongoing. The performance of the charge measurement by means of the time over threshold method has been demonstrated. Calibration measurements to the prepared and tested successfully.

\section{References}

[1] ATLAS Pixel Collaboration, ATLAS Pixel Detector Technical Design Report, CERN/LHCC/98-13 (1998).

[2] G. Aad et al., The ATLAS Experiment at the CERN Large Hadron Collider, JINST 3 (2008) P08003.

[3] G. Aad et al., ATLAS Pixel Detector Electronics and Sensors, JINST 3 (2008) P07007.

[4] Commissioning of the ATLAS Pixel Optical Readout Link, ATLAS Note (to be published).

[5] Threshold Tuning of the ATLAS Pixel Detector, ATLAS Note (to be published). 